\title{
MoBaS on Phosphorylation Data
}

\author{
Marzieh Ayati \\ Case Western Reserve \\ University \\ 10900 Euclid Ave \\ Cleveland, $\mathrm{OH}$ \\ mxa401@case.edu \\ Goutham Narla \\ Case Western Reserve \\ University \\ 10900 Euclid Ave \\ Cleveland, $\mathrm{OH}$ \\ gxn71@case.edu
}

\author{
Danica Wiredja \\ Case Western Reserve \\ University \\ 10900 Euclid Ave \\ Cleveland, $\mathrm{OH}$ \\ ddw34@case.edu \\ Mark R Chance \\ Case Western Reserve \\ University \\ 10900 Euclid Ave \\ Cleveland, $\mathrm{OH}$ \\ mrc16@case.edu
}

\author{
Daniela Schlatzer \\ Case Western Reserve \\ University \\ 10900 Euclid Ave \\ Cleveland, $\mathrm{OH}$ \\ dms73@case.edu \\ Mehmet Koyuturk \\ Case Western Reserve \\ University \\ 10900 Euclid Ave \\ Cleveland, $\mathrm{OH}$ \\ mxk331@case.edu
}

\begin{abstract}
Although advances in high-throughput omics technologies revolutionized our understanding of the genomic underpinnings of cancer, there are still many challenges in understanding how patients with common driver mutations may display diverging phosphoproteomic responses to the same treatment. Thus, an examination of the signaling landscape will provide essential molecular information for modeling personalized patient treatment design. However, integrative bioinformatics approaches to identify phosphoproteomicsbased molecular states are in their infancy. To address this challenge, we integrated several bioinformatics tools to compare and contrast the drug-induced global signaling alterations of two KRAS mutated non-small cell lung cancer (NSCLC) cell lines, A549 and H358, treated with a novel activator of the tumor suppressor Protein Phosphatase 2A (PP2A) versus DMSO control.

To identify protein subnetworks that are enriched in differentially phosphorylated proteins, we use our algorithm $\mathrm{MoBaS}$ which is designed to identify protein subnetworks that are enriched in disease-associated genomic variants identified by genome-wide association studies (GWAS). MoBaS takes as input a PPI network and a score for each protein indicating the protein's association with the phenotype of interest. It then identifies protein subnetworks that are (i) composed of densely interacting proteins, and (ii) enriched in proteins with high scores. MoBaS also assesses the statistical significance of the identified subnetworks using permutation tests that enable multiple hypothesis testing. Subsequently, we do kinase enrichment within the statistical significant modules and find Aurora KB as a key kinase differentially regulated between the two cell lines in response to our compound. Further corroborating this finding, Au-
\end{abstract}

rora $\mathrm{KB}$ was downregulated at the protein and mRNA levels with our treatment in A549 but not in H358. Ultimately, our approach models the diverging protein-level drug response across two similar cell lines representing NSCLC patients. Such phosphoproteomic information will potentially inform the optimal therapy regimen for each individual.

\section{Categories and Subject Descriptors}

J.3 [Life and Medical Sciences]: Biology and genetics

\section{General Terms}

Algorithms, Design, Experimentation

\section{Keywords}

Phosphoproteomics, protein protein interaction networks, functional modules, statistical significance, network analysis
Permission to make digital or hard copies of part or all of this work for personal or classroom use is granted without fee provided that copies are not made or distributed for profit or commercial advantage, and that copies bear this notice and the full citation on the first page. Copyrights for third-party components of this work must be honored. For all other uses, contact the owner/author(s). Copyright is held by the author/owner(s).

$B C B^{\prime} 16$, October 2-5, 2016, Seattle, WA, USA.

ACM 978-1-4503-4225-4/16/10.

http://dx.doi.org/10.1145/2975167.2995267. 